\title{
Current research in aging: a report from the 2015 Ageing Summit
}

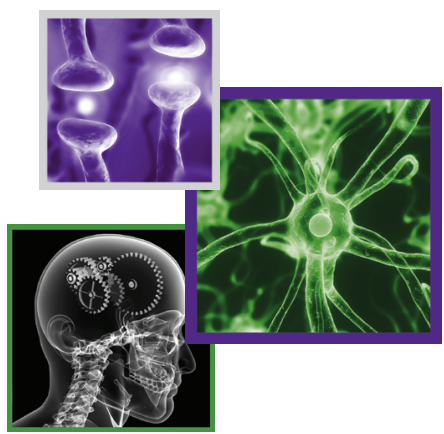

\author{
Emmanuel Moyse $e^{*, 1,2}$, Lies Lahousse $^{3,4}$ \& Slavica Krantic ${ }^{5}$
}

\section{Ageing Summit, London, UK, 10-12 February 2015}

\begin{abstract}
SUMMARY The Ageing Summit 2015 held on 10-12 February 2015 in London (UK) provided an extensive update to our knowledge of the 'Biology of Ageing' and a forum to discuss the participants' latest research progress. The meeting was subdivided into four thematic sessions: cellular level research including the aging brain; slowing down progression, rejuvenation and self-repair; genetic and epigenetic regulation; and expression and pathology of age-related diseases. Each session included multiple key presentations, three to five short research communications and ongoing poster presentations. The meeting provided an exciting multidisciplinary overview of the aging process from cellular and molecular mechanisms to medico-social aspects of human aging.
\end{abstract}

Ken Parkinson (Queen Mary University of London, UK) opened the meeting with the first among nine recognized hallmarks of aging [1]: the universal tumor-suppressive process of replicative senescence. As initially identified in vitro on fibroblasts, it leads to an original phenotype including permanent cell cycle arrest, telomere shortening and dysfunction, unrepairable DNA double strand breaks, alteration of cell secretions (senescence-associated secretory phenotype) and a metabolic shift away from the Krebs cycle toward glycolysis and the pentosephosphate pathway [2]. Comparative biochemical analysis of conditioned media from senescent versus quiescent and proliferating cultures revealed 30 specifically accumulated metabolites in senescence (among the 261 detected), over half of which also accumulate within human chronological aging and five of which are represented in a recently published signature of human aging and associated indicators of health. One of the metabolites that accumulated was kynurenine and this has been associated with Alzheimer's disease in humans.

The presentation by David Granville (viDA Therapeutics, Inc., Canada) touched upon another hallmark of aging [1]: chronic inflammation, and resulting extracellular matrix degradation. A major mediator in chronic inflammatory diseases is the serine protease granzyme B. Its causal role in skin aging acceleration by ultraviolet light, for example, a case of extrinsic tissue aging was demonstrated by using a granzyme $B$ knockout mouse [3]. Relevant to neurology, granzyme B deficiency also protects against diet-induced aging of Apolipoprotein E-knockout mice, via blockade of arterial wall thinning by inflammatory mediators.
'PRC Laboratory, University François Rabelais/Centre INRA of Tours, 37380 Nouzilly, France

${ }^{2}$ Cordeliers Faculty of Medicine, University Paris-Descartes, 15 rue de l'Ecole de Médecine, 75006 Paris, France

${ }^{3}$ Ghent University Hospital, De Pintelaan 185, 9000 Ghent, Belgium

${ }^{4}$ Erasmus Medical Center, PO Box 2040, 3000 CA Rotterdam, The Netherlands

${ }^{5}$ Cordeliers Research Centre, INSERM-Paris-Descartes-University, 15 rue de l'Ecole de Médecine, 75006 Paris, France

*Author for correspondence: emmanuel.moyse@univ-tours.fr

\section{KEYWORDS}

- cellular neurobiology of aging $\bullet$ microglia $\bullet$ oxidative stress $\bullet$ proteostasis $\bullet$ sensory organs $\bullet$ stem cells - telomerase • vascular dysfunction 
Another hallmark of aging [1]: alterations in mitochondria function, were focus of Hideo Tsukada's (Hamamatsu Photonics KK, Japan) presentation on the brain mitochondrial complex-I activity with PET-scan studies in adult monkeys using the radioprobe $\left[{ }^{18} \mathrm{~F}\right] \mathrm{BCPP}-\mathrm{EF}$. As a methodological validation, this tool detected the well-established age-related reduction of brain metabolism. It was then applied to a brain ischemia model and facilitated the detection of a PET signal decrease 1 day postlesion and increase after 7 days, which corresponded respectively to neuronal damage at the subacute phase of stroke and to the delayed perilesional inflammatory microglial activation.

The role of immune cells in brain aging was presented by Jennifer Pocock (Institute of Neurology, UK). Microglia represent $16 \%$ of the total human adult CNS cells. Their number drops by healthy aging and rises after neurological lesions. During aging, the microglial phenotype changes from the neuroprotective 'M1' to the dystrophic senescent neuroinflammatory 'M2', which may represent different types of microglia rather than plasticity [4]. Adult microglia evolve into primed microglia within the aging CNS, with MHC-II and Ibal as markers and the increased secretion of proinflammatory cytokines. Oxidative stress is a hallmark of aging [1] which results from transcriptional induction of pro-oxidant genes and represses growth and antioxidant gene expression. It leads to microglial activation, which activates cell stress pathways and triggers microglia loss by apoptosis. The current challenge is to identify the substances released by microglia which might damage neurons.

The loss of proteostasis and altered intercellular communications as hallmarks of aging [1] were also discussed. Cheryl Frye (University of Alaska, Canada) focussed on the relationship between hormones and aging, more specifically on the role of neurosteroids in the aging brain. Sex steroids can indeed influence cognitive functions, especially progesterone via its potent agonist properties at GABA-A and NMDA receptors. Increasing evidence shows that progesterone and its brain-derived metabolites stimulate some memory aspects in aged rodents, while low levels are observed in the brain of murine Alzheimer models.

Naji Tabet (Brighton \& Sussex Medical School, UK) discussed pharmaceutical tools to slow down the progression of Alzheimer's disease since no disease-modifying treatment exists to date. The most efficient drugs (e.g., acetylcholine neurotransmission enhancers like donepezil) may initially slow the disease progression, but after a given time, cognitive decline further accelerates and the beneficial effects of pharmaceutical treatments are quickly lost. Researchers now hope to make progress in this field by targeting risk factors such as obesity, hypertension, diabetes, poor diet, physical inactivity, among others. Several clinical trials for disease modifying agents are currently underway.

George Kuchel (University of Connecticut, CT, USA) presented his view on the human 'frailty' concept that has been coined by modern geriatrics as it involves both stochastic (random) events and specific phenotypes. Frailty was also later on defined by Lies Lahousse (Ghent University Hospital, Belgium) as the disability to compensate function loss. Frail elderly people have more falls, hospitalizations and an increased mortality risk beyond their age, sex and comorbidities [5]. In addition to the brain, endocrine and immune systems, skeletal muscle is involved in the development of frailty [6]. Concerning the latter, Professor Kuchel shared observations that muscle performance typically declines more than does muscle mass in the elderly. It remains to be seen whether muscle atrophy and degeneration are associated with frailty.

Anton JM Roks (Erasmus Medical Center, The Netherlands) presented novel data about the genomic instability underlying age-related dysfunction of vascular endothelium in mice [7]. Altered biological parameters included hypertension, vascular stiffness, vascular smooth muscle cell dysfunction, decreased endothelial nitric oxide synthase, cell senescence, cumulative DNA damages and an increased DNA repair response. Changes in phosphodiesterases appear to play a central role in the vascular dysfunction caused by genomic instability, and diet restriction promises to be an effective treatment.

Gabriele Saretzki (Institute for Aging and Health, UK) discussed the role of the telomerase protein TERT in brain aging and neurodegeneration. After updating the biochemical structure of ubiquitous telomerase, she reviewed its multiple functions besides its canonical role in telomere extension. In vitro experimental TERT overexpression suppresses oxidative stress, mtDNA damage and apoptosis [8]. In vivo, telomerase activity in the mouse brain is down-regulated quickly during development, and TERT decreases during aging while reactive oxygen species increase. Lack of TERT augments oxidative stress in mouse brain and decreases memory in old mice; 
reciprocally, TERT is increased by $30-40 \%$ in mouse brain mitochondria by aging-improving interventions like dietary restriction or rapamycin treatment, and TERT overexpression improves memory. TERT is expressed in neurons and activated microglia of rodent and human brains, but is absent from astrocytes. In the human brain, TERT is maintained through Alzheimer's disease progression and increases in the hippocampus at Braak's stage VI, while TERT and neurotoxic phosphorylated TAU protein mutually exclude each other within one cell. This suggests a protective role for TERT against neurodegeneration [9].

The relevance of the DNA-damage hallmark of aging [1] for the brain was addressed by Adalberto Merighi (University of Turin, Italy). aging-related alterations of chromatin structure, cell cycle length and histone code in the brain were reviewed including the case of neural stem cells. The phospho-H2AX histone component of DNA damage response machinery was pointed as a senescence marker in the mouse brain.

Another hallmark of aging, stem cell exhaustion [1], was examined by Richard Richardson (McGill University, Canada). Domination of cell proliferation over cell death drives carcinogenesis, whereas aging is characterized by reduced stem cell proliferation and increased cell death. Quantitative analysis of published data concerning approximately 31 human organs revealed greater organ involution and aging in highly proliferative tissues, indicating adaptability of replicative senescence [10].

Maeve Rea (Queens University Belfast, UK) revealed some early results from the European Genetics of Healthy Aging project [11]. Supported by evidence from the nonagenarian BELFAST cohort, aging genes involved in longevity can be classified into specific cellular functions: stress response, hormone biosynthesis, inflammatory and immunological responses, mitochondrial functioning, metabolism.

The classical oxidative stress theory of aging was rejuvenated by Kelvin JA Davies (University of Southern California, CA, USA) in his talk on declining stress responses during aging. Stress responses are adaptive, for example, transient, cellular reactions which involve variations in gene expression. Proteasome efficiency is increased within $24 \mathrm{~h}$ of acute oxidative stress onset through the selective induction of some of its subunits. A universal and evolutionary conserved mediator of numerous stress responses at the cellular level is the Nrf2 transcription factor. With regard to aging, oxidative damage starts accumulating only in the last third of life; implicating no gradual process. Senescence is correlated with increased levels of carbonylated proteins and decreased proteasome subunit inducibility.

Majd AlGhatrif (National Institute on Aging, MD, USA) discussed arterial aging as a potential cause of inadequate control of hypertension with aging. The hallmark of arterial aging is isolated systolic hypertension resulting from widened pulse frequency. The role of the aorta as a capacitor to minimize pulsatility by shifting energy from systole to diastole (Windkessel effect) is progressively impaired with age because of increased stiffness and diameter of aortic wall [12]. The one size fits all strategy to treat hypertension is suboptimal due to individual variation in the age-associated alterations in the properties underlying a given blood pressure value. Better characterization of individual aortic biomechanical and hemodynamic properties to guide individualized therapy appears the most effective to achieve blood pressure control.

Age-related hearing loss was presented by Varela-Nieto (Instituto de Investigaciones Biomédicas 'Alberto Sols', Spain) in relation with a detrimental role of IGF-1 message weakening with mammalian aging. The molecular bases for this phenomenon were discussed and included increased inflammation and deregulation of autophagy. The clinical success of IGF-1 medication to improve hearing in sudden hearing loss patients supports this observation in mice [13].

Jeremy M Sivak (University of Toronto, Canada) presented the aging eye as a case of detrimental disbalance among multiple intercellular signals. The inner retina provides a neurovascular system which is particularly vulnerable to aging processes. The transcriptional coactivator PGC-1a is established as a master homeostasis regulator of inner retina and impacts several key targets of aging: mitochondria biogenesis, vascular regulation, antioxidant responses. Retina content of PGC-1a increases with age in rodents. In contrast with glaucoma models, PGC-1a decreases in retina with age. Glaucoma is an age-related, neurodegenerative disease of the retina that impacts ganglion cell neurons, for which no cure exists. PGC-1a knockout mice show increased vulnerability of retinal ganglion neurons, whereas PGC-1a stimulates antioxidant activity of retinal astrocytes [14]. This presentation along with many others during this meeting, increased our knowledge on the cellular 
and genetic mechanisms of aging and provided promising new targets for strategies to improve a healthy life span.

Euroscicon's Ageing 2016 event will take place from 9 to 11 February 2016 [15].

Financial \& competing interests disclosure

The present work was supported by the french Foundation

for Medical Research (FRM, grant no. DVS20131228910 to S Krantic) and by the Fund for Scientific Research Flanders (FWO, grant no. G035014N to L Lahousse). The authors have no other relevant affiliations or financial involvement with any organization or entity with a financial interest in or financial conflict with the subject matter or materials discussed in the manuscript apart from those disclosed.

No writing assistance was utilized in the production of this manuscript.

\section{References}

1 Lopez-Otin C, Blasco MA, Partridge L, Serrano M, Kroemer G. The hallmarks of aging. Cell 153, 1194-1217 (2013).

2 James EL, Michalek RD, Pitiyage GN et al. Senescent human fibroblasts show increased glycolysis and redox homeostasis with extracellular metabolomes that overlap with those of irreparable DNA damage, aging, and disease. J. Proteome Res. 14(4), 1854-1871 (2015).

3 Parkinson LG, Toro A, Zhao $\mathrm{H}$ et al. Granzyme b mediates both direct and indirect cleavage of extracellular matrix in skin after chronic low-dose ultraviolet light irradiation. Aging Cell 14, 67-77 (2015).

4 Streit WJ, Xue QS, Tischer J, Bechmann I. Microglial pathology. Acta Neuropathol. Commun. 2, 142 (2014).

5 Lahousse L, Maes B, Ziere G et al. Adverse outcomes of frailty in the elderly: the rotterdam study. Eur. J. Epidemiol. 29, 419-427 (2014).
6 Clegg A, Young J, Iliffe S, Rikkert MO, Rockwood K. Frailty in elderly people. Lancet 381, 752-762 (2013).

7 Durik M, Kavousi M, van der Pluijm I et al. Nucleotide excision DNA repair is associated with age-related vascular dysfunction. Circulation 126, 468-478 (2012).

8 Saretzki G. Extra-telomeric functions of human telomerase: cancer, mitochondria and oxidative stress. Curr. Pharm. Des. 20, 6386-6403 (2014).

9 Spilsbury A, Miwa S, Attems J, Saretzki G. The role of telomerase protein tert in alzheimer's disease and in tau-related pathology in vitro. J. Neurosci. 35, 1659-1674 (2015).

10 Richardson RB, Allan DS, Le Y. Greater organ involution in highly proliferative tissues associated with the early onset and acceleration of ageing in humans. Exp. Gerontol. 55, 80-91 (2014).
11 Deelen J, Beekman M, Uh HW et al. Genome-wide association meta-analysis of human longevity identifies a novel locus conferring survival beyond 90 years of age. Hum. Mol. Genet. 23, 4420-4432 (2014).

12 Scuteri A, Morrell CH, Orru M et al. Longitudinal perspective on the conundrum of central arterial stiffness, blood pressure, and aging. Hypertension 64, 1219-1227 (2014).

13 Varela-Nieto I, Murillo-Cuesta S, Rodriguezde-la-Rosa L et al. IGF-I deficiency and hearing loss: molecular clues and clinical implications. J. Pediatr. Endocrinol. Rev. 10(4), 460-472 (2013).

14 Guo X, Dason ES, Zanon-Moreno V et al. PGC-1 $\alpha$ signaling coordinates susceptibility to metabolic and oxidative injury in the inner retina. Am. J. Pathol. 184, 1017-1029 (2014).

15 Ageing 2016. www.regonline.co.uk/Ageing2016 